\title{
Coherence and predictability of extreme events in irregular waves
}

\author{
A. L. Latifah ${ }^{1}$ and E. van Groesen ${ }^{1,2}$ \\ ${ }^{1}$ Applied Mathematics, University of Twente, The Netherlands \\ ${ }^{2}$ Labmath-Indonesia, Bandung, Indonesia \\ Correspondence to: A. L. Latifah (a.1.latifah@utwente.nl)
}

Received: 16 January 2012 - Revised: 1 March 2012 - Accepted: 14 March 2012 - Published: 26 March 2012

\begin{abstract}
This paper concerns the description and the predictability of a freak event when at a certain position information in the form of a time signal is given. The prediction will use the phase information for an estimate of the position and time of the occurrence of a large wave, and to predict the measure of phase coherence at the estimated focussing position. The coherence and the spectrum will determine an estimate for the amplitude. After adjusting for second order nonlinear effects, together this then provides an estimate of the form of a possible freak wave in the time signal, which will be described by a pseudo-maximal signal. In the exceptional case of a fully coherent signal, it can be described well by a so-called maximal signal.

We give four cases of freak waves for which we compare results of predictions with available measured (and simulated) results by nonlinear AB-equation (van Groesen and Andonowati, 2007; van Groesen et al., 2010). The first case deals with dispersive focussing, for which all phases are (designed to be) very coherent at position and time of focussing; this wave is nearly a maximal wave. The second case is the Draupner wave, for which the signal turns out to be recorded very close to its maximal wave height. It is less coherent but can be described in a good approximation as a pseudo-maximal wave. The last two cases are irregular waves which were measured at MARIN (Maritime Research Institute Netherlands); in a time trace of more than 1000 waves freak-like waves appeared "accidentally". Although the highest wave is less coherent than the other two cases, this maximal crest can still be approximated by a pseudo-maximal wave.
\end{abstract}

\section{Introduction}

In this paper we consider extreme waves that can "accidentally" appear in irregular, uni-directional wave fields with very broad spectrum and relatively low value of the
Benjamin-Feir index (BFI). These waves satisfy the common definition (Dysthe et al., 2008; Slunyaev et al., 2005; Kharif and Pelinovsky, 2003) of rogue, or freak, wave that the wave height exceeds two times the significant wave height. However, different from much current research on rogue waves, the modulational instability does not play a (dominant) role. Instead of nonlinearly dominated waves, the extreme waves here will appear at position and time of a high degree of coherence, in the sense that many wave components contribute to a linearly dominated constructive interference phenomenon. This agrees with Gemmrich and Garrett (2008) that many extreme waves are merely the simple consequence of linear superposition. For realistic wind waves, this coherence may be just as important as nonlinear effects (which may have played a role to obtain the coherence, and may enforce the linear converging of group lines near the extreme event). In fact, we will show that the well-known Draupner (or New Year) wave (Haver, 2004), measured in the NorthSea, shows a high degree of coherence while its BFI of approximately 0.55 (Janssen, 2003; Adcock and Taylor, 2009c) is below the critical threshold value 1 . In addition, we will show similar extreme waves that were generated accidentally in experiments on irregular waves in a wave tank at MARIN hydrodynamic laboratory. In two experiments and successive numerical calculations, with in total more than 2300 waves that were observed evolving downstream above a flat bottom over a distance of at least 30 wavelengths, 3 of such rogue events could be identified. Measurements and numerical simulations show a relatively gradual growth and decay before and after the rogue event. This long-life character does not satisfy the other characterization of rogue waves that these should appear suddenly and disappear quickly. Also, as has also been shown for four other measured freak events in the North Sea (Slunyaev et al., 2005), the linear and nonlinear simulations show remarkable little difference in shape and wave height, although with some difference of position and time due to nonlinear effects in propagation speed. 
Referring to wave tank experiments by Shemer et al. (2010); Shemer and Sergeeva (2009), it should be noticed that these experiments were designed to study BFIdominated rogue waves. In these experiments, narrow band Jonswap spectra with $\gamma=7$ and some narrow Gaussian spectra are considered. The coherence reported in (Onorato et al., 2006; Shukla et al., 2006) refers to the phase coupling between free wave and the higher order bound waves due to nonlinear wave generation. But since the free waves have random phase, these experiments can be seen as a bridge between the pure "soliton" (Akhmediev-breather; Akhmediev et al., 2011) rogue waves that generate a triangle spectrum shape from an initially very narrow spectrum, and the low valued BFI irregular waves (obtained for broad band Jonswap spectra with $\gamma=3$ ) as will be described here.

In this paper we will characterize and discuss in various ways the appearance of extreme events and the role played by coherence as a constructive interference property. By this is meant that the phase nearly vanishes for waves in a considerable interval around the peak frequency. Together with the almost linear evolution property, this fact makes it possible to design a prediction method for this type of rogue waves. We will show that from a given elevation signal measured at some observation point, the position, time and profile of the rogue event can be rather well predicted over distances of 30 or more wave lengths. The method searches for the freak event by looking for the position and time such that the total phase, obtained by linear evolution of the observed phase, has minimal variance. Supported by linear and nonlinear numerical simulations and experimental observations, the predictions of rogue events for the Draupner and for the MARIN waves will be investigated and compared.

The contents of the paper can be described as follows. Sections 2 and 3 deal with the effect of partial or complete constructive interference. In Sect. 2 we consider time signals, obtained when vanishing phases in a so-called maximal wave create fully constructive interference at a certain instant. For a Jonswap spectrum as example, the effect of partial interference is investigated. For fixed random phase $\theta(\omega) \in(-\pi, \pi]$, signals with a fraction of that phase, so phase $\alpha \theta(\omega) \in \alpha(-\pi, \pi]$, are investigated for increasing $\alpha \in[0,1]$. Upon increasing $\alpha$ until for $\alpha=1$ the irregular signal (fully random) is obtained, the highest elevation in the maximal signal will decrease while the background grows, with clusters of larger and smaller waves depending on $\alpha$. The details of the full signal depend on the choice of the initial phase function $\theta$, but the average over random phases for fixed $\alpha$, produces a so-called pseudo-maximal wave, which is shown to be a scaled version of the maximal wave, with scaling amplitude tending to zero for $\alpha \rightarrow 1$.

In Sect. 3 we show the corresponding process for linear waves, and investigate the effects of 2 nd order nonlinear Stokes contributions (detailed formulas are given in Appendix A). The linear propagation modifies the phase with $K(\omega) x$ where $x$ is the displacement, and $K(\omega)$ the wave number related to $\omega$. The nonlinear contributions, for realistic cases of wind waves in a coastal area, change the spectrum. But the changes are mainly in the long-wave components (leading to wave set-down) and slightly in the higher components but mainly in a neighbourhood of the double peak frequency, as expected. The nonlinear effects on the maximal wave are small, and just as well for the irregular wave, except for some different propagation speed.

In line with these observations, we formulate in Sect. 4 the prediction method based on the minimization of the phase variance over time and space. And we describe in detail 4 study cases; after a specially designed experiment for dispersive focussing, we investigate the Draupner wave and two irregular MARIN waves. The prediction method is shown to be capable to detect the extreme waves reasonably well.

In the final Sect. 5 we conclude with some additional remarks and conclusions.

\section{Signal coherence: from maximal to irregular signals}

\subsection{Notation}

Since waves in the ocean are described at each point by a time signal, we first consider real valued signals with zero mean defined on a time interval $[0, T]$. We introduce some notation, and then derive a priori estimates for the highest possible wave heights. In the following we describe the relation between a function $s(t)$ and its Fourier transform $\check{s}(\omega)$ using notation with integrals as

$s(t)=\int_{-\omega_{\max }}^{\omega_{\max }} \check{s}(\omega) e^{-i \omega t} d \omega$ and $\check{s}(\omega)=\frac{1}{2 \pi} \int_{0}^{T} s(t) e^{i \omega t} d t$

Here $\omega_{\max }=2 \pi / \Delta t$ and $2 \pi / T=\Delta \omega$ will be used because in practical situations we deal with discrete signals sampled with some time step $\Delta t$. From the real-valuedness of the signal we have $\breve{s}(\omega)=\check{s}(-\omega)$ (the bar denoting complex conjugation) and for the phase $\theta(\omega)=-\theta(-\omega)$. Hence

$$
\begin{aligned}
s(t) & =\int_{-\omega_{\max }}^{\omega_{\max }} \check{s}(\omega) e^{-i \omega t} d \omega=\int_{-\omega_{\max }}^{\omega_{\max }}|\check{s}(\omega)| e^{i \theta(\omega)} e^{-i \omega t} d \omega \\
& =2 \int_{0}^{\omega_{\max }}|\check{s}(\omega)| \cos (\theta(\omega)-\omega t) d \omega
\end{aligned}
$$

Parceval's identity links the $L_{2}-$ norms of the signal and its FT:

$\int_{0}^{T} s^{2}(t) d t=2 \pi \int_{-\omega_{\max }}^{\omega_{\max }}|\check{s}(\omega)|^{2} d \omega=4 \pi \int_{0}^{\omega_{\max }}|\check{s}(\omega)|^{2} d \omega$

We define the variance and standard deviation $\sigma$ of the signal,

$$
\begin{aligned}
\sigma^{2} & =\operatorname{Var}(s)=\frac{1}{T} \int_{0}^{T} s^{2}(t) d t \\
& =\frac{4 \pi}{T} \int_{0}^{\omega_{\max }}|\check{s}(\omega)|^{2} d \omega=2 \Delta \omega \int_{0}^{\omega_{\max }}|\check{s}(\omega)|^{2} d \omega
\end{aligned}
$$


the significant wave height $H_{s}$ as $H_{s}=4 \sigma$, the (one-sided) spectrum $E(\omega)$ such that

$\int_{0}^{\omega_{\max }} E(\omega) d \omega=\operatorname{var}(s)$,

so $E(\omega)=2 \triangle \omega|\check{s}(\omega)|^{2}=\frac{4 \pi}{T}|\check{s}(\omega)|^{2}$, and higher order moments

$m_{n}=\int_{0}^{\omega_{\max }} \omega^{n} E(\omega) d \omega$

\subsection{Maximal signal}

From

$$
\begin{aligned}
|s(t)| & =2\left|\int_{0}^{\omega_{\max }}\right| \check{s}(\omega)|\cos (\theta(\omega)-\omega t) d \omega| \\
& \leq \int_{-\omega_{\max }}^{\omega_{\max }}|\check{s}(\omega)| d \omega
\end{aligned}
$$

it is seen that the inequality is actually an equality if at some time the cosine is identically 1 . This can happen (only) if the total phase $\phi(\omega)=\theta(\omega)-\omega t$ vanishes for all $\omega$ at that time, say at $t=T_{\text {foc }}$. Then the signal has its maximal possible value:

$\max _{t} s(t)=s\left(T_{\mathrm{foc}}\right)=\int|\check{s}(\omega)| d \omega$ if $\theta(\omega)-\omega T_{\mathrm{foc}}=0$

For this reason we will call a signal with all phases zero at some time a maximal signal,

$s_{\max }=\int_{-\omega_{\max }}^{\omega_{\max }}|\check{s}(\omega)| \cos \left(\omega\left(t-T_{\mathrm{foc}}\right)\right) d \omega ;$

at $T_{\text {foc }}$ all wave components contribute to a constructive interference. We will show maximal signals for a spectrum given by a Jonswap spectrum that is commonly used to describe developing wind wave fields. The specific expression is given by

$$
\begin{aligned}
E_{\mathrm{Jon}}(\omega) & =A g^{2}\left(\frac{\omega_{p}}{\omega}\right)^{5} \exp \left(-\frac{5}{4}\left(\frac{\omega_{p}}{\omega}\right)^{4}\right) \gamma^{r}, \\
r & =\exp \left[-\frac{1}{2 \varsigma^{2}}\left(\frac{\omega}{\omega_{p}}-1\right)^{2}\right]
\end{aligned}
$$

The parameter $\gamma$ specifies the narrow bandedness of the spectrum; the choice $\gamma=3$ is taken for most realistic coastal situations and provides a broad band spectrum. Meanwhile the parameter $A$ is related to the wave amplitude. We took as illustration $A=0.0408$; these values are motivated by study cases of irregular MARIN waves in Sect. 4.2. The $\varsigma$ is defined as $\varsigma=0.007$ if $\omega \leq \omega_{p}$ and $\varsigma=0.009$ if $\omega>\omega_{p}$. In Fig. 1 the dotted, solid and dashed line corresponds with $\gamma=1.5, \gamma=3$, and $\gamma=7$ respectively (the narrow spectrum for $\gamma=7$ was used in Shemer et al., 2010).

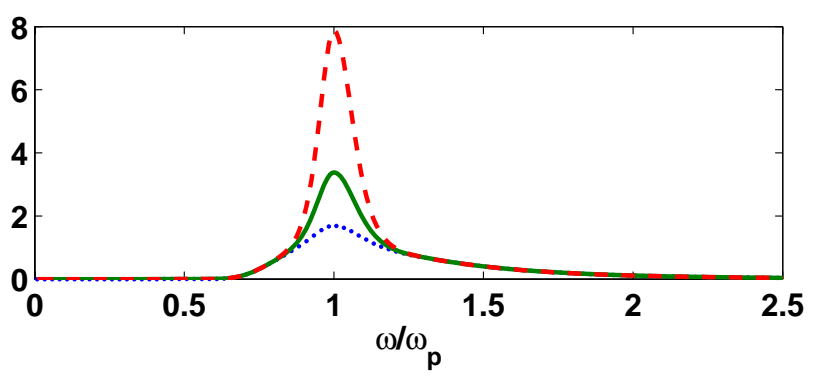

Fig. 1. The Jonswap spectrum, $E_{\mathrm{J} o n}(\omega)$, where $A=0.0408$ and $\gamma=1.5$ (dotted), $\gamma=3$ (solid), $\gamma=7$ (dashed).

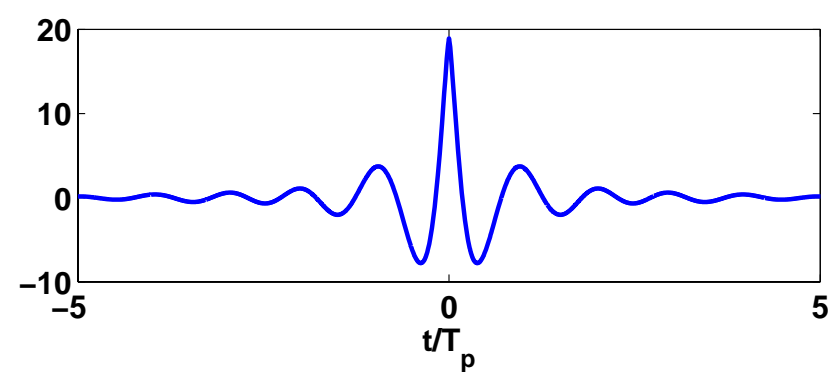

Fig. 2. The maximal signal corresponding to Jonswap spectrum with $\gamma=3$.

In order to see the maximal crest height of a wave with the Jonswap spectrum, we give an example for $\gamma=3$. The plot of the maximal signal of Jonswap spectrum for $\gamma=3$ is given in Fig. 2. This maximal signal has significant wave height about $3.4 \mathrm{~m}$ and the maximal possible amplitude is approximately $19 \mathrm{~m}$. From Fig. 2 we can see that the wave is confined to an interval of length equal to 8 peak periods. Outside the interval the wave nearly vanishes.

\subsection{Phase effects}

For the maximal signal above, all phases vanish at one position. In this section we investigate the effect of non-vanishing phases which may be partly coherent or random.

An irregular signal is obtained in case the phases are uniformly distributed in $(-\pi, \pi]$. To investigate cases in between a completely random signal and a fully coherent maximal signal, we will consider signals with 'cut-off' phases. That is, for given random function $\theta(\omega) \in(-\pi, \pi]$, we consider for $\alpha \in[0,1]$ signals with phase $\theta_{\alpha}=\alpha \theta$. Although not much can be said about an individual signal, the ensemble averaged signal at fixed $\alpha$, denoted by

$$
\begin{aligned}
{[s]_{\alpha} } & =\text { Average } \int_{-\omega_{\max }}^{\omega_{\max }}|\check{s}(\omega)| \cos \left(\theta_{\alpha}(\omega)-\omega\left(t-T_{\mathrm{foc}}\right)\right) d \omega \mid, \\
\theta_{\alpha} & \in \alpha U(-\pi, \pi)
\end{aligned}
$$

is interesting. Using the Strong Law Large Number (Ross, 2007) it can be shown that this average is a scaled version 
For $\alpha=0.6$
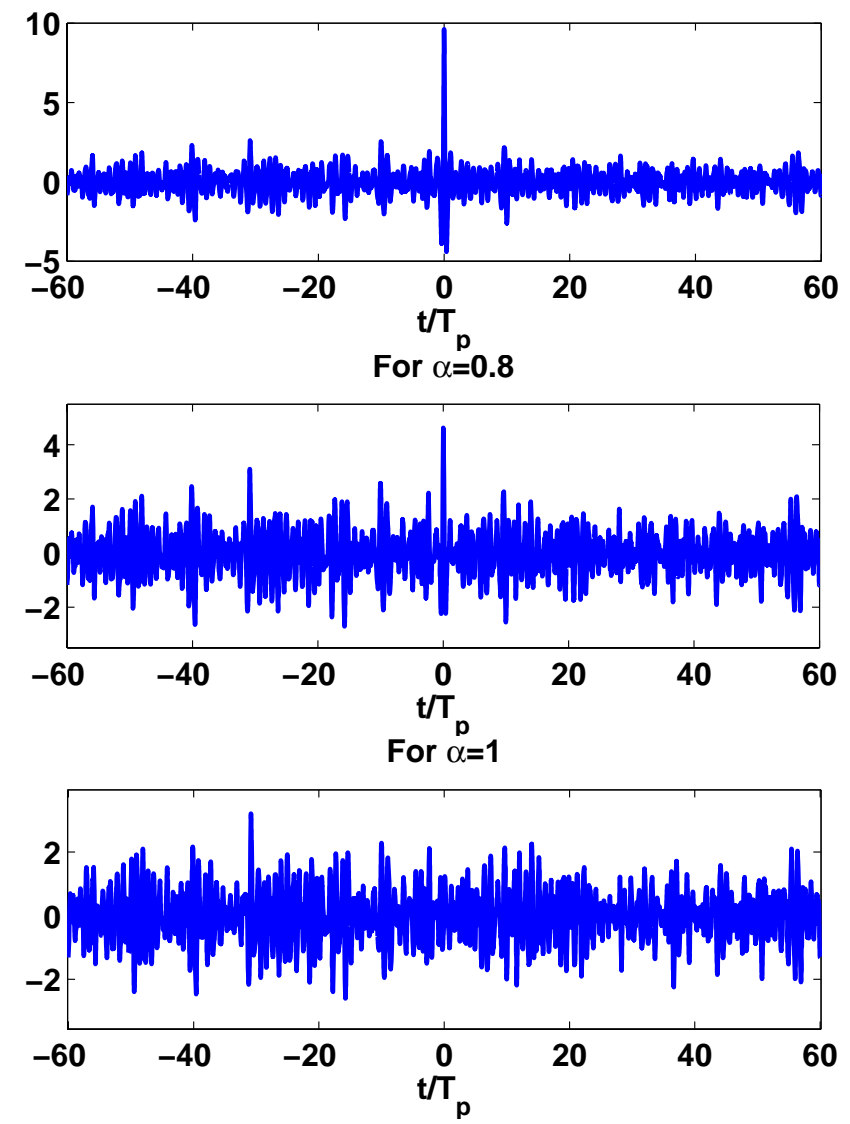

Fig. 3. Jonswap signal with significant wave height of $3.4 \mathrm{~m}$ and random phases in $\alpha(-\pi, \pi]$ in which $\alpha=0.6, \alpha=0.8$, and $\alpha=1$.

of the maximal signal. We will call this average a pseudomaximal signal; it can be written as

$[s]_{\alpha}=\rho(\alpha) s_{\max }$

where the scaling factor is

$\rho(\alpha)=\frac{\sin (\alpha \pi)}{\alpha \pi}$

For identically vanishing phases, the maximal signal was already shown in Fig. 2. In the plots of Fig. 3 we show for a given Jonswap spectrum $E_{\mathrm{Jon}}(\omega)$ with $\gamma=3$, the effect of phases. For a fixed random phase $\theta(\omega) \in(-\pi, \pi]$, we illustrate the effect of adding a fraction of that phase $\alpha \theta(\omega) \in \alpha(-\pi, \pi]$, for increasing $\alpha \in[0,1]$. Upon increasing $\alpha$, the extreme wave is decreasing while the background grows. The original extreme wave may disappear completely, while in the background clusters of larger and smaller waves are formed, depending on $\alpha$ and on the specific random function $\theta(\omega)$; characteristic effects are visible in Fig. 3 .

\section{Wave coherence and pm-waves}

In this section we illustrate for synthetic cases that wave coherence plays an important role in the appearance of extreme events in irregular wave trains. Extreme events will appear at instants and positions of a high degree of coherence, to be defined precisely in the following. This will prepare for the examples in the next section, and will motivate the prediction method of freak waves.

Furthermore, we will show by investigating the evolution over longer periods and positions, that away from the focussing area, the wave has still a considerable amplitude over a long range. Stated differently, the extreme wave is not an isolated phenomenon on an almost flat sea, but builds up gradually and disappears gradually back into the background. Since these phenomena are observed in linear as well as in nonlinear irregular waves, we will investigate effects of nonlinearity, effects on the spectrum as well on the wave evolution.

\subsection{Pseudo-max waves}

A wave evolution in 1-D denoted by the surface elevation $\eta(x, t)$ describes at each position $x$ the signal $t \rightarrow \eta(x, t)$. In fact, for a given elevation signal $s_{\mathrm{obs}}(t)$ at one observation point $X_{\text {obs }}$, we can describe the uni-directional linear evolution as

$\eta(x, t)=\int\left|\check{s}_{\text {obs }}(\omega)\right| \cos (\Phi(t, x, \omega)) d \omega$

where $\Phi(t, x, \omega)=K(\omega)\left(x-X_{\text {obs }}\right)+\theta_{\text {obs }}(\omega)-\omega t$ is the total wave phase, $K(\omega)$ is the wave number related to the frequency by the dispersion relation. For exact dispersion of infinitesimal waves, $K$ is the inverse of $\Omega$ given by

$\Omega(k)=\operatorname{sign}(k) \sqrt{g k \tanh (k D)}$

where $g$ is the gravitational acceleration and $D$ is the water depth.

We determine the focussing position and time $\left(X_{\text {foc }}, T_{\text {foc }}\right)$ at which the phase variance

$$
\begin{aligned}
P V(x, t) & =\operatorname{Var}(\Phi(t, x, \omega)) \\
& =\int_{0}^{\omega_{\max }}|\Phi(t, x, \omega)|^{2} d \omega
\end{aligned}
$$

is minimal, so-called $\mathrm{PV}_{\text {foc }}$. In practice we compute the phase variance over an interval of the dominant frequencies $\left[\omega_{\min }, \omega_{\max }\right]$. We define $\Gamma$ as the degree of coherence,

$\Gamma=1-P V_{\text {foc }}$.

For given random phase $\theta_{\alpha}=\alpha \theta$ as described in the pseudo-maximal signal from Sect. 2.3, the phase variance can be computed to be $\operatorname{PV}\left(\theta_{\alpha}\right)=(\alpha \pi)^{2} / 3$. Conversely, for 
arbitrary phases, we will take this relation to define $\alpha$ to correspond with the phase variance. In particular at focussing we define

$\alpha_{\text {foc }}^{2}=\frac{3}{\pi^{2}} \mathrm{PV}_{\text {foc }}$

and then define a pseudo-maximal (pm) wave as

$\eta_{\mathrm{pm}}(x, t)=\rho\left(\alpha_{\mathrm{foc}}\right) \int\left|\check{s}_{\mathrm{obs}}(\omega)\right| \cos (\Phi(t, x, \omega)) d \omega$

with total wave phase $\Phi(t, x, \omega)=K(\omega)\left(x-X_{\text {foc }}\right)-$ $\omega\left(t-T_{\text {foc }}\right)$. This pseudo-max wave will model the neighbourhood of the extreme event.

Figure 4 shows the density plots for the linear evolution of Jonswap waves with a restricted random phase for $\alpha=0,0.6,0.8$, and 1 . Besides that, we present the density of the variance of the total wave phase. Those density plots are shown in a frame moving with the group velocity. From both densities we can observe the position and the propagation of the wave with $\alpha=0$ or $\alpha=0.6$; the development of the high wave into the focussing wave is noticeable and the position of the minimal phase variance $(\mathrm{PV})$ which shows the focussing position is prominent. The case with $\alpha=0.8$ does not show the high waves clearly and the position of the focussing is hardly visible. For $\alpha=1$, the Jonswap signal is purely random and there is no clear extreme wave.

\subsection{Nonlinear effects}

In this section we will take into account the nonlinear wave contributions, therefore we can investigate the importance of nonlinearity, especially in some cases we study here. From laboratory observation, a focussing signal has nonzero phase at low frequencies; there is a generated nonlinear interaction which causes a nonlinear set-down contribution. Moreover, a second order set-up contribution might appear. The effect of the nonlinear interaction should be involved as suggested by Clamond and Grue (2002), especially for highly-nonlinear phenomenon of freak wave. Therefore a nonlinear pm-wave needs to be designed to describe an extremal wave profile more precisely.

A nonlinear pm-wave will now be defined by adding the second-order contributions to the linear pm-wave; we neglect the higher order contributions. The quadratic nonlinear interaction of two waves with frequencies $\omega_{1}$ and $\omega_{2}$ produces higher-order waves with possible frequencies of $0,2 \omega_{1}, 2 \omega_{1}$, $\omega_{1}+\omega_{2}$, and $\omega_{1}-\omega_{2}$. The general interactions for pair of waves have been given by Dalzell (1999). For the irregular waves we are dealing with, we sum up all the two waves interactions. The full expression of the nonlinear pm-wave is then given by:

$\eta_{\mathrm{pm}}(x, t)=\rho\left(\alpha_{\mathrm{foc}}\right)\left(I_{01}+I_{02}+I_{p}+I_{m}\right)$

$$
\begin{aligned}
I_{01} & =\int|\check{s}(\omega)| \cos (\Phi(x, t)) d \omega \\
I_{02} & =\int|\check{s}(\omega)|^{2}\left(B_{0}(k)+B_{2}(k) \cos (2 \Phi(x, t))\right) d \omega \\
I_{p} & =\iint\left|\check{s}\left(\omega_{2}\right) \check{s}\left(\omega_{2}\right)\right| B_{p}\left(k_{1}, k_{2}\right) \cos \left(\Phi_{1}+\Phi_{2}\right) d \omega_{1} d \omega_{2} \\
I_{m} & =\iint\left|\check{s}\left(\omega_{2}\right) \check{s}\left(\omega_{2}\right)\right| B_{m}\left(k_{1}, k_{2}\right) \cos \left(\Phi_{1}-\Phi_{2}\right) d \omega_{1} d \omega_{2},
\end{aligned}
$$

where $\Phi(x, t)=K(\omega)\left(x-X_{\text {foc }}\right)-\omega\left(t-T_{\text {foc }}\right)$ and the coefficients $B_{0}, B_{2}, B_{p}$, and $B_{m}$ are symmetric functions of $K(\omega)$ defined in Appendix A. The first term is the linear pmwave defined in Eq. (8). $I_{02}$ is the contribution generated by two identical frequencies. $I_{p}$ and $I_{m}$ are the contributions of two different frequencies; $I_{m}$ gives a set-down contribution. According to (Chen, 2006) this set-down contribution is actually much more significant than the classical Stokes term. This set-down makes it possible for a nonlinear wave to have a lower amplitude than the linear wave. The effect of the second-order contributions will be shown in Jonswap spectrum case in Sect. 3.2.1. The linear part is a so-called "free" wave, meaning that the wave number and frequency satisfy the dispersion relation. The other quadratic waves are so-called bound waves: the sum or difference of the wave numbers and the corresponding frequencies do not satisfy the dispersion relation and hence would not satisfy individually the wave equations, but their "bounded" connection with the constituent free waves does satisfy the law of wave propagation.

The maximal signal corresponding to a given time signal is symmetric in time around the time of focussing $T_{\text {foc }}$. Since a pseudo-maximal signal is just a scaled version of a maximal signal, the same holds true for a pseudo-maximal signal. Similarly, a (linear) maximal and a pm-wave is symmetric in time around $T_{\text {foc }}$, and just as well symmetric in space around position $X_{\text {foc }}$. From the nonlinear interactions shown above, it follows that even nonlinear corrections will respect these symmetry properties.

\subsubsection{Effects on spectrum}

A Jonswap spectrum is a spectrum that is supposed to describe realistic random sea waves, thereby neglecting long waves. Consequently the spectrum contains contributions from free and bound waves. To see the contribution of the bound waves, we plot in Fig. 5 an example of a Jonswap spectrum (solid). Then we construct and plot the spectrum obtained by removing the second order bound waves and long waves (dashed line), so-called free-wave Jonswap. The subtraction of bound waves changes the original Jonswap spectrum by short wave removal that starts at 1.65 times the peak frequency, and annihilates practically all waves above $2 \omega_{p}$. If we then add second order nonlinear contributions to this free-wave spectum, we almost precisely recover the original Jonswap spectrum; a small overshoot near $2.3 \omega_{p}$ is 

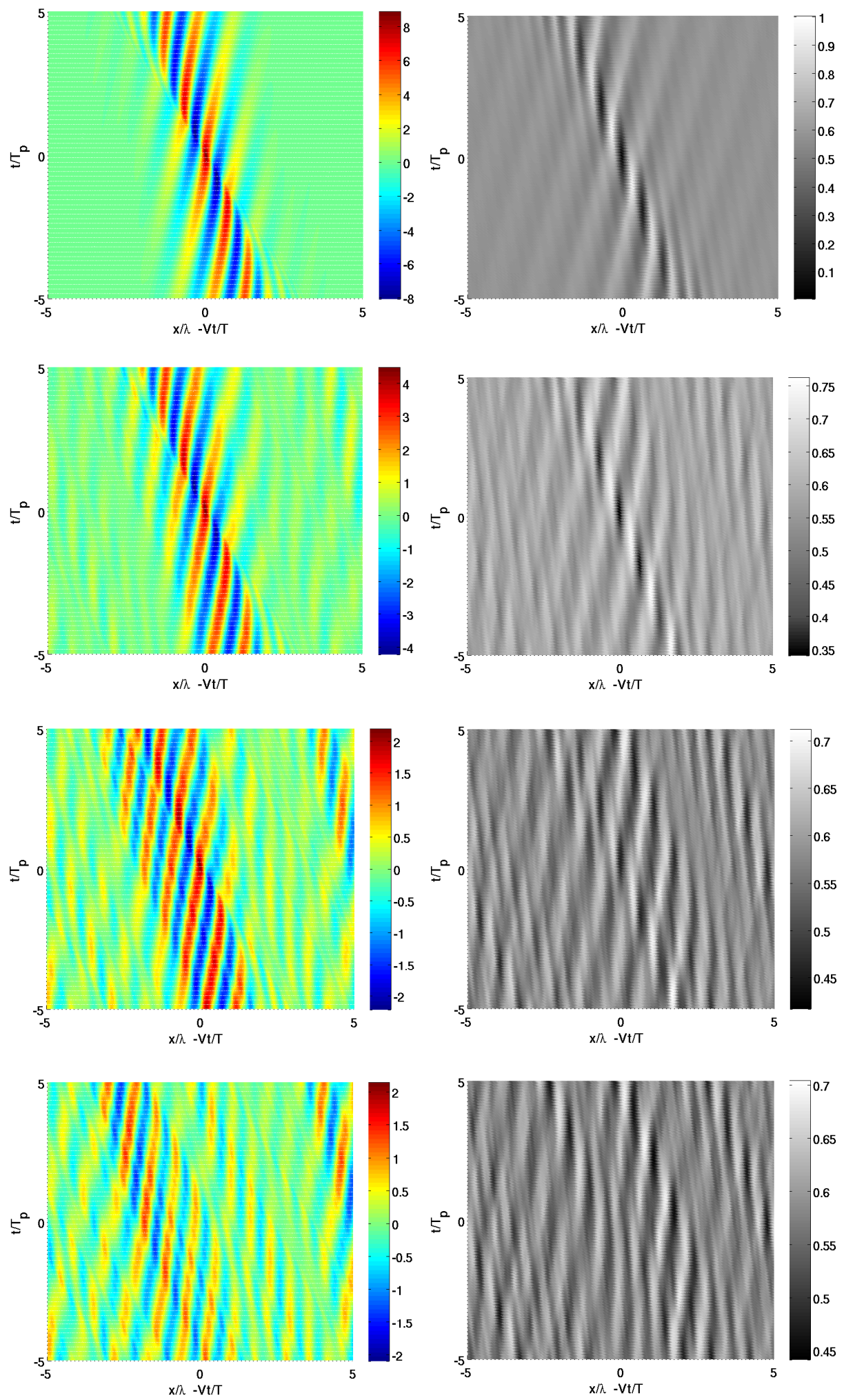

Fig. 4. In successive rows we show plots of the linear evolution of Jonswap waves with a random phase restricted for $\alpha=0,0.6,0.8$ and 1 , respectively. At the left density plots are shown of the evolution in a frame moving with the group velocity (horizontal axis, time vertical axis with normalized units). At the right, with the same axis the evolution of the density of the phase variance is shown. 

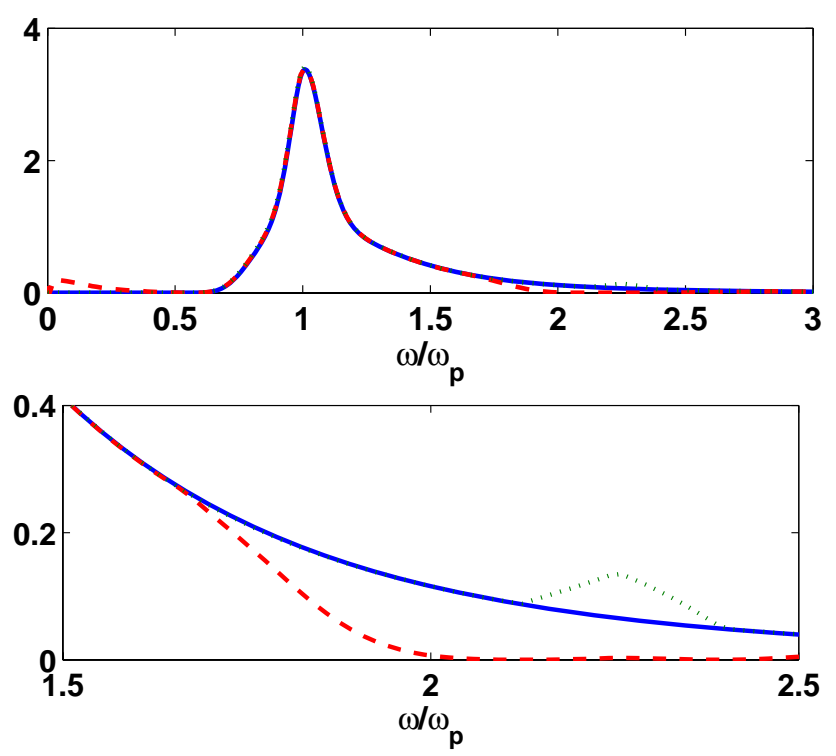

Fig. 5. Top: The original Jonswap spectrum (solid), the free-wave Jonswap spectrum without bound waves (dashed), and the freewave spectrum with nonlinear contributions (dotted). Bottom: The zoomed version.

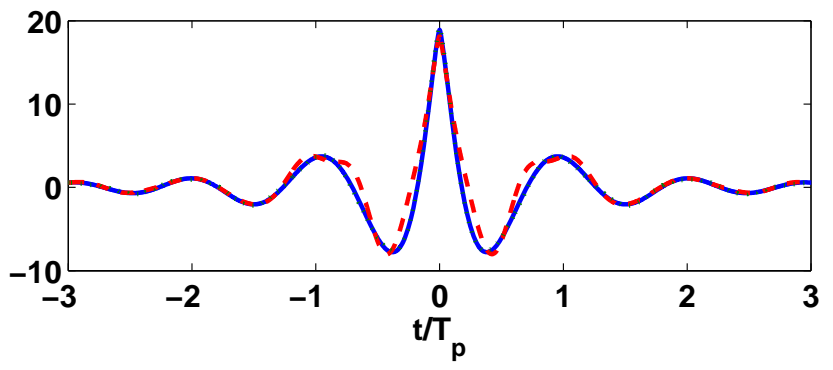

Fig. 6. The maximal signals corresponding to the original Jonswap spectrum (solid), the free-wave spectrum (dashed) and corresponding to the free-wave spectrum with nonlinear contributions (dotted, behind the solid line).

the only difference. This is visible in the enlarged lower plot in Fig. 5.

To investigate the nonlinear effects on the signals, we consider the maximal signals corresponding to the 3 spectra above. In Fig. 6 we plotted the maximal signal of the original Jonswap (solid), the maximal signal of the free-wave Jonswap (dashed) and the maximal signal of the free-wave spectrum with the nonlinear additions (dotted, invisible behind the solid line). The plots show that the high frequency contributions make only little difference for the maximal signal.

\subsubsection{Effects on wave evolution}

In this section we present the nonlinear wave evolution of the four cases of Jonswap signal as shown in Fig. 7. In the extreme case of the Jonswap signal with zero phases, the difference of the linear (see Fig. 4) and nonlinear evolution shows itself mainly in the propagation speed and wave height. The highest amplitude in the nonlinear evolution appears earlier than in the linear evolution; a similar behaviour is seen for the case of the Jonswap signal with random phases in $0.6(-\pi, \pi]$.

For Jonswap signal with $\alpha=0$ which is a maximal wave, the density plot of the nonlinear evolution is not as smooth as the linear evolution. Around the focussing position and for $\alpha=0.6$ we can observe the symmetry of the waves. The amplitude of the nonlinear wave is higher than the linear wave, as can be observed from the color bar in Fig. 7. For Jonswap signal with $\alpha=0.8$ or $\alpha=1$ which is mostly random, the difference between linear and nonlinear evolution is more difficult to see.

\section{Freak wave prediction method and study cases}

The description in the previous section leads to a simple and direct strategy to make predictions of the highest wave that will occur during the wave evolution. In the first subsection we describe the method for linear dispersive evolution to which we will restrict. This strategy will then be applied in four study cases in Sect. 4.2.

\subsection{Linear Prediction method}

Starting point is a given time signal $s_{\mathrm{obs}}(t)$ at a given position $X_{\text {obs. }}$. The length of the time interval of the signal is essential; despite some dispersive broadening of that interval while evolving away from $X_{\mathrm{obs}}$, predictions can only be made within this (with distance shifted) time interval.

From the phase information of $s_{\text {obs }}(t)$ we determine the variance of the total wave phase, and look at its minimal value in space and time, finding $\left(X_{\mathrm{foc}}, T_{\mathrm{foc}}\right), P V_{\mathrm{foc}}$ and the coherence $\Gamma_{\text {foc }}$. Using the spectrum of $s_{\text {obs }}(t)$, and calculating the phase band $\alpha_{\text {foc }}$ related to $\Gamma_{\text {foc }}$, we obtain the pseudomaximal wave with parameter $\alpha_{\text {foc }}$.

It will be shown in the study cases that this pm-wave will approximate the highest wave that occurs in the linear wave evolution from the observed signal $\eta(x, t)$ in a neighbourhood of $\left(X_{\mathrm{foc}}, T_{\mathrm{foc}}\right)$; in particular $\left(X_{\mathrm{foc}}, T_{\mathrm{foc}}\right)$ estimates the position and time of the appearance of this highest wave. But also the shape of the time signal at $X_{\mathrm{foc}}: t \rightarrow \eta\left(X_{\mathrm{foc}}, t\right)$ for times near $T_{\text {foc }}$ will be well approximated by the pseudomaximal signal. We can actually reconstruct a more reliable signal prediction $\eta_{\text {foc }}$ which is the signal of the linear wave evolution at position $X_{\text {foc }}$ :

$\eta_{\text {foc }}(t)=\int|\check{s}(\omega)| \cos \left(\Phi\left(t-T_{\text {foc }}, X_{\text {foc }}, \omega\right)\right) d \omega$ 

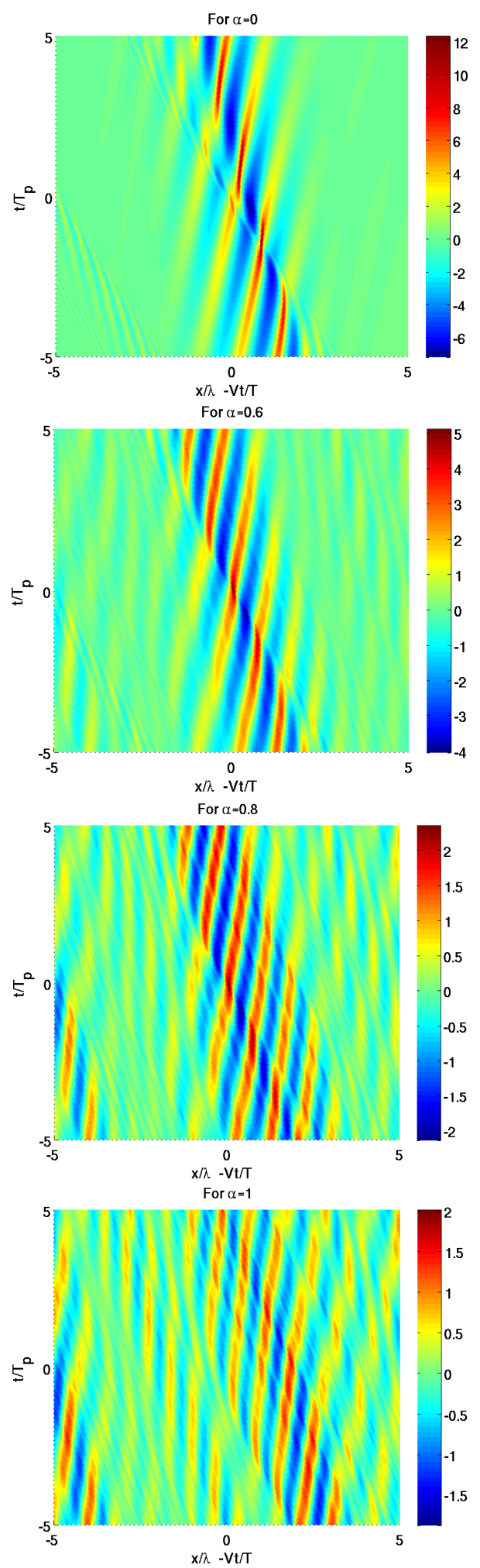

Fig. 7. Similar as Fig. 4 left column, but now for nonlinear evolution, the waves with Jonswap spectrum and restricted phase $\alpha(-\pi, \pi]$ with $\alpha=0,0.6,0.8$ and 1 .

In the following we will compare predictions with numerical simulations. Although the numerical results are not crucial for the main results presented here, we use linear and nonlinear simulations to compare with the linear-based predictions. When we talk about linear simulations in the following, this refers to simulations for the linear evolution with the exact dispersion relation (using a spectral method). Simulations with the AB-model refer to a nonlinear spectral code that has been described in various publications (van Groesen and Andonowati, 2007; van Groesen et al., 2010; van Groesen and Andonowati, 2011). Specifically, in (van Groesen and van der Kroon, 2012) the freak wave of the study case IW12 and in (van Groesen et al., 2011) the freak wave of the study case IW9 have been described in detail.

\subsection{Study cases}

The four study cases, for which measurements are available to test our descriptions and predictions of appearance of freak waves, are a dispersive focussing wave in a wave tank, MARIN experiment 202002, the Draupner wave with elevation measurement obtained from Sverre Haver of Statoil, and two irregular waves of Jonswap type, which were measured at MARIN but scaled (1:50 in space) to geophysical dimensions. For the irregular waves, the first case is IW12, Marin experiment 103001 with peak period $12 \mathrm{~s}$ and the second case is IW9, Marin experiment 102003 with peak period $9 \mathrm{~s}$.

For each case we follow the same strategy and show plots to illustrate the findings, which are summarized in a conclusive table at the end. From elevation heights at a certain position $X_{\text {obs }}$ we predict the pseudo-maximal wave: its coherence $\Gamma$, the position $X_{\text {foc }}$ and time $T_{\text {foc }}$ of focussing, and determine from that the maximal crest height and the maximal wave height at the moment of focussing. For the Draupner wave and the irregular waves we also provide the significant wave height and the Benjamin Feir index BFI as calculated at $X_{\mathrm{foc}}$. The BFI is a measure of the quotient of nonlinearity and spectrum width. Various versions were described in the literature (Shemer, 2010); we will use the definition from Janssen (2003), BFI $=\frac{\sqrt{2} \epsilon}{\Delta \omega / \omega_{p}}$. Since we are dealing with deterministic waves, we define the nonlinearity $\epsilon$ by $k a_{\mathrm{rms}}\left(a_{\mathrm{rms}}\right.$ is the root mean square amplitude) as suggested in (Kharif et al., 2009; Osborne et al., 2005; Slunyaev, 2006). The spectral width $\triangle \omega$ is defined according to the energy level that corresponds to half of the spectral peak value (Shemer, 2010) and $\omega_{p}$ is the peak frequency.

\subsubsection{Dispersive focussing}

This first study case is a designed wave at MARIN based on the principle of dispersive wave focussing. The maximal wave height is more than five times the highest waves at the generation position. As we will show, at time and position of focussing, the wave is almost perfectly a maximal wave that can be accurately predicted from the initial signal at the 


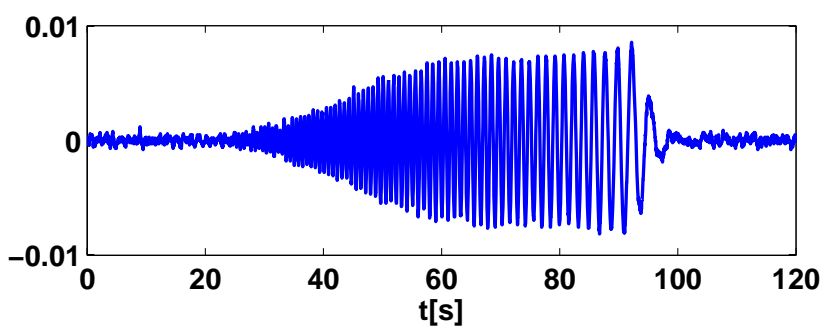

Fig. 8. Measured time signal at $X_{\mathrm{obs}}=10 \mathrm{~m}$ of the focussing wave.

Table 1. Parameters for the dispersive focussing wave.

\begin{tabular}{lllll}
\hline CASE & \multicolumn{2}{l}{ Focussing wave } & & \\
$\begin{array}{l}\text { Depth } \\
\text { Position } X_{\text {obs }}\end{array}$ & $\begin{array}{l}1 \mathrm{~m} \\
10 \mathrm{~m}\end{array}$ & & & \\
& Prediction & \multicolumn{2}{l}{ Simulation } & Meas \\
\hline Meas position & & linear & $\mathrm{AB}$ & 50 \\
$X_{\text {foc }}$ & 50.05 & 50.1 & 50.2 & \\
$T_{\text {foc }}$ & 109.3 & 109.34 & 109.4 & 109.3 \\
$\Gamma$ (coherence) & 1 & 0.99 & 0.99 & 0.99 \\
Max Crest height & 0.061 & 0.053 & 0.057 & 0.055 \\
Max Waveheight & 0.086 & 0.081 & 0.081 & 0.08 \\
\hline
\end{tabular}

observation position. In this case, the observation position is $X_{\mathrm{obs}}=10 \mathrm{~m}$. The initial signal is shown in Fig. 8 .

First we compute the coherence by minimizing the variance of the total wave phase. Using spectrum and phase information of the initial signal, the variance of the total wave phase by choosing $\omega_{\min }=1.26$ and $\omega_{\max }=8.85$ is found to be minimal for $T_{\text {foc }}=109.3$ and $X_{\text {foc }}=50.05$; the minimal value is $P V_{\text {foc }}=0.001$; the value of coherence $\Gamma$ is 0.999 (nearly fully coherent). Thus the extremal wave profile at focussing can be described well by a maximal signal.

The left Fig. 9 shows the linear and nonlinear maximal signal; the right one compares the time signal of the $\mathrm{AB}$ simulation result at focussing and the nonlinear maximal signal (the highest crests are fitted at $t=109.4 \mathrm{~s}$ ), including the spectrum and the phase. We use the spectrum and the phase to show the differences caused by nonlinear effect. In this case the nonlinear correction does not significantly affect the amplitude of the linear maximal signal. The effect of adding the second-order nonlinear corrections is almost invisible. The right Fig. 9 shows that the nonlinear maximal signal follows the actual focussing behaviour and perfectly models the extremal wave profile at focussing. For both low frequencies and high frequencies the spectrum is lifted up similar to the actual evolution by AB-simulation, although a bit lower. By observing the phase, we know the nonlinear correction in maximal signal yields long waves set down with phase $\pi$ or $-\pi$ in about $\omega<1$ as in the actual evolution.

The minimal variance of the total wave phase is obtained at $(50.05 ; 109.3)$ : the linear prediction leads to a focussing point at $x=50.05 \mathrm{~m}$ and focussing time of $t=109.3 \mathrm{~s}$. This can be seen from Fig. 10 showing the density plot of the phase variance as a function of $x$ and $t$ : the minimal phase variance is quite well visible since the area of minimal phase variance is very small and is surrounded by much higher values. To validate the predicted focussing position, the actual evolution is calculated by nonlinear AB simulation. The validation at the precise focussing position could not be done by measurement because of the limited number of the measured positions, but the nearby measurement at $50 \mathrm{~m}$ confirms the simulation results. Some other results of the AB-simulation are shown in Fig. 11. In the nonlinear $A B$ simulation for which the density of the evolution is shown in the lower plot of Fig. 11, the extreme wave occurs at $x=50.2 \mathrm{~m}$ with the time focussing at $t=109.4 \mathrm{~s}$. The upper plot of Fig. 11 presents the maximal temporal amplitude (the highest amplitude at each position during the time evolution) of the linear and nonlinear evolution by AB model, showing that the linear and nonlinear $\mathrm{AB}$ simulations do not differ significantly. The detailed comparison is presented in Table 1, confirming that the linear prediction agrees very well with the nonlinear evolution.

\subsubsection{Draupner wave}

The Draupner wave (also called New Year wave) is a point measurement at approximately $70 \mathrm{~m}$ depth under the Draupner platform (16/11-E) in the North Sea off the coast of Norway. The measurement of this time signal is $20 \mathrm{~min}$ long. We will first show that the wave shape at the Draupner position $X_{\mathrm{Dr}}$ is well approximated by a pm-signal by adjusting the height to the observed crest height.

To compare the Draupner wave with a pm-wave, we observe that the maximal wave corresponding to the spectrum would have crest height $37.5 \mathrm{~m}$, instead of the actual height of $18.5 \mathrm{~m}$. The ratio $18.5 / 37.5=0.49$ is taken as multiplication factor of the maximal wave, which is precisely a pmwave with $\alpha=0.6$ and coherence $\Gamma=0.88$. The plots of the Draupner wave (solid) and the shifted pm-wave (dashed) are shown in Fig. 13.

Using the observed signal at $X_{\operatorname{Dr}}$ we predict that actually an even higher wave has occurred at a few meters distance. In the following we take for convenience $X_{\mathrm{Dr}}=0$. To test prediction capacity over longer distances, we simulate a backward (nonlinear) evolution to a synthetic observation position $X_{\text {synth }}=X_{\operatorname{Dr}}-400$, and use the (nonlinearly corrected) linear prediction method to determine the pm-wave from the signal information at $X_{\text {synth }}$.

To predict from $X_{\text {synth }}$ the position of the extreme wave, the minimal value of the phase variance is computed; Fig. 15 shows the density plot of the phase variance. In this case, we restrict the frequencies to calculate the phase variance to the interval $\omega \in(0.25 ; 1)$ as the linear wave contribution seems to be dominant in this interval. Then the minimal value of the phase variance is $\mathrm{PV}_{\text {foc }}=0.12$, which leads to $\alpha=0.6$. This 

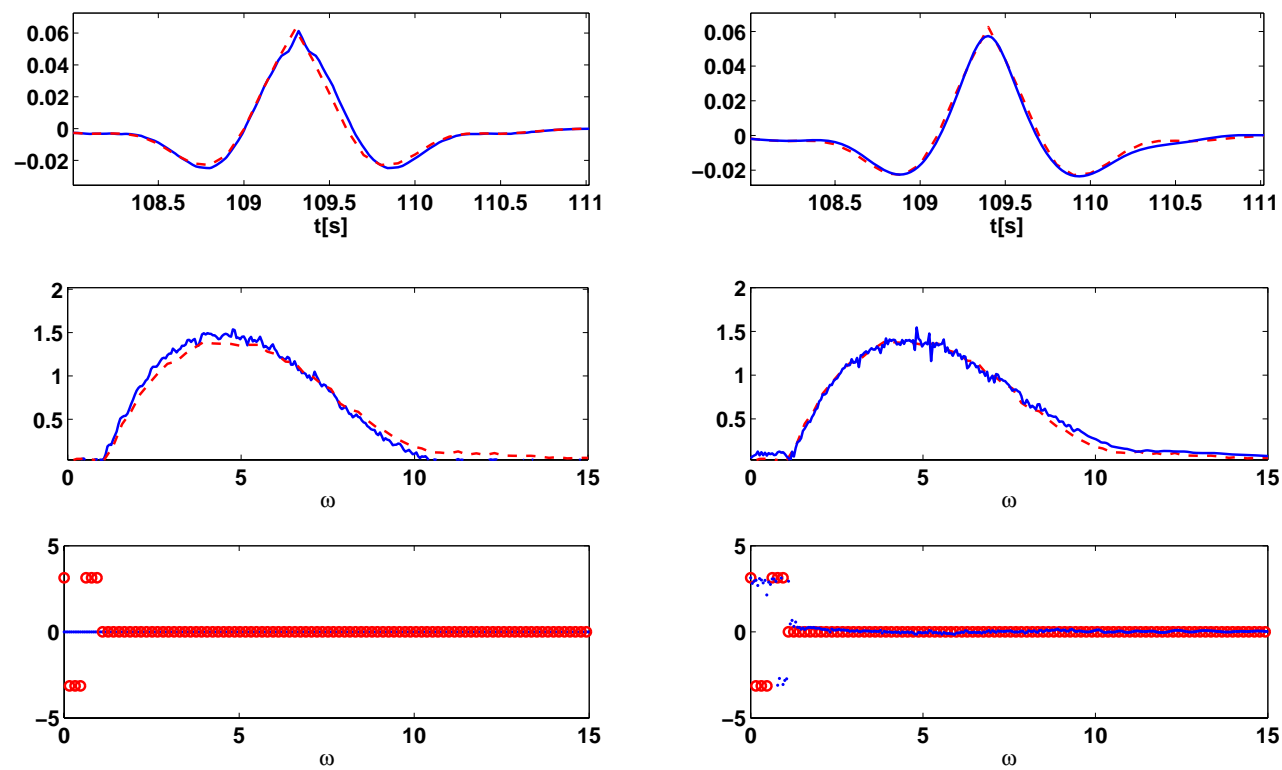

Fig. 9. We show in the left column the comparison between properties for the linear (solid) and the nonlinear maximal signal (dashed), and in the right column a comparison between properties of the nonlinear maximal signal (solid) and AB-simulations started at $X=10 \mathrm{~m}$ of the signal at $X=50.2 \mathrm{~m}$ (dashed). In the upper row for the signals, in the middle row for the spectrum, and in the lower row for the phase.

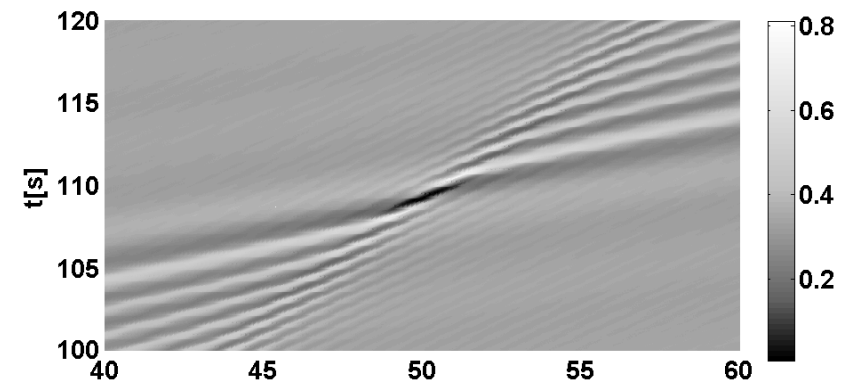

Fig. 10. The zoomed density plot of the variance of the total wave phase $\mathrm{PV}(x, t)$. The minimal value $\mathrm{PV}_{\text {foc }}$ is shown in black.

value of $\alpha$ is related to a pseudo-maximal wave with scaling factor of 0.5 .

With the linear prediction, the most coherent wave is found at $x=9 \mathrm{~m}$ and $t=1.1 \mathrm{~s}$ which is shown in Fig. 13, approximately the position of the Draupner wave with $1.1 \mathrm{~s}$ shifted. The plot of the signal prediction from $X_{\text {synth }}$ is also shown in Fig. 13. Table 2 provides parameters of the prediction and the $\mathrm{AB}$ evolution using the initial time signal at $X_{\text {synth }}$, and of the measurement.
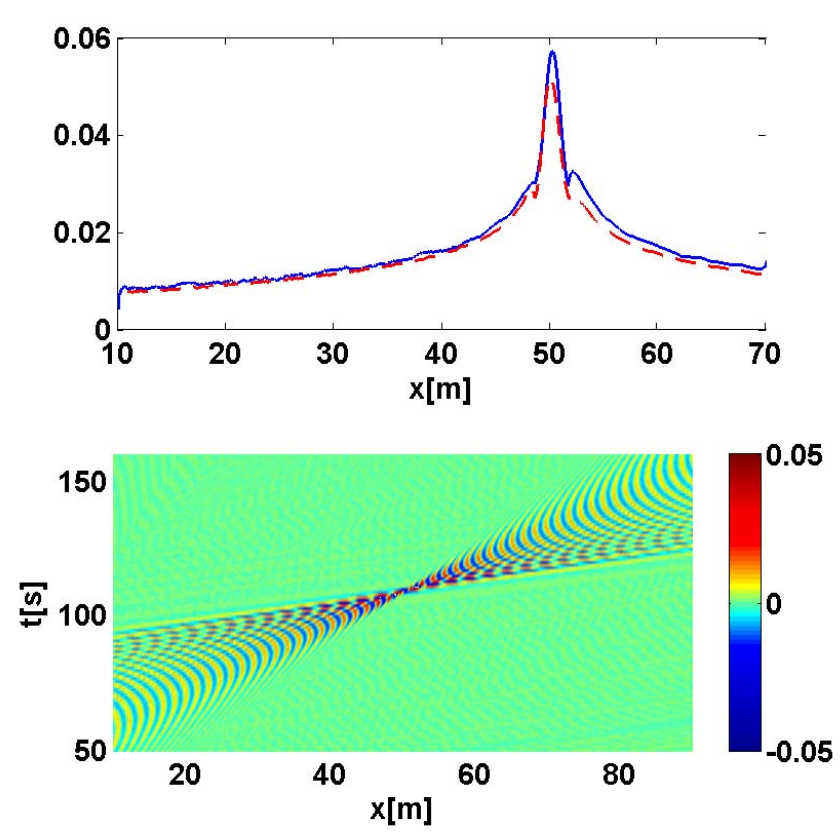

Fig. 11. Top: Maximal Temporal Amplitude of linear (dashed) and nonlinear (solid) AB simulation; Bottom: Density plot of the nonlinear $\mathrm{AB}$ simulation. 


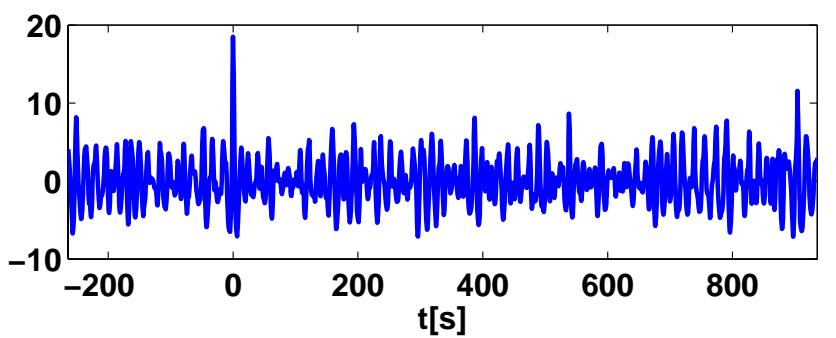

Fig. 12. The Draupner signal, with time of highest wave crest put at $t=0$

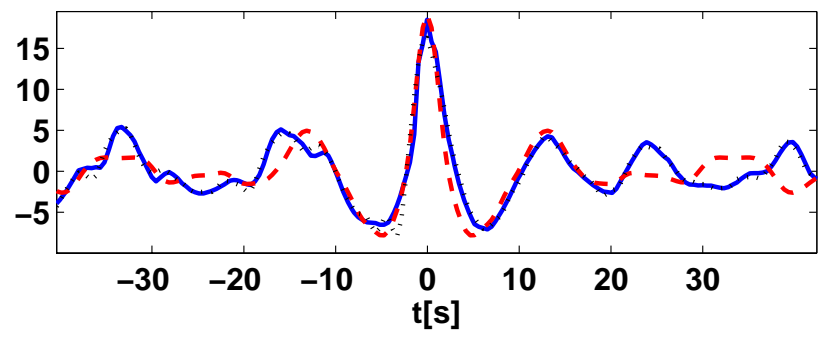

Fig. 13. Draupner Wave (solid), Pm-signal (dashed), and Signal prediction (dotted line) which has been shifted so that the highest peak is at $t=0$.

\subsubsection{Irregular waves}

The last two study cases of irregular waves provide a more realistic situation than the Jonswap example treated in Sect. 3. Although there are measurement positions more or less close to the highest wave appearance, we used simulations to compare the prediction results and compare wave shapes at the focussing.

The examples presented here show that irregular wave can generate freak events. In the laboratory experiment the freak wave appeared accidentally in a time record of about $30 \mathrm{~min}$. Actually, at the end of the tank there was a 1:20 slope to study coastal effects, but we will restrict here to the waves above the flat part of the tank; reflections from the slope (and tank boundaries) were small and not relevant for our considerations.

In our description below, the dimensions and results are scaled to a geophysical situation with a spatial factor of 50 , and corresponding temporal factor of $\sqrt{50}$.

\subsubsection{Irregular wave IW12}

We use as initial time signal the surface elevation of an irregular wave as measured $39.15 \mathrm{~m}$ from the wave maker in the wave tank, similar to $1957.7 \mathrm{~m}$ in geophysical dimension. Further on we always use the geophysical dimension. The initial time signal has total length of more than $3 \mathrm{~h}$, with significant wave height of $3.14 \mathrm{~m}$ and is shown in Fig. 16. We

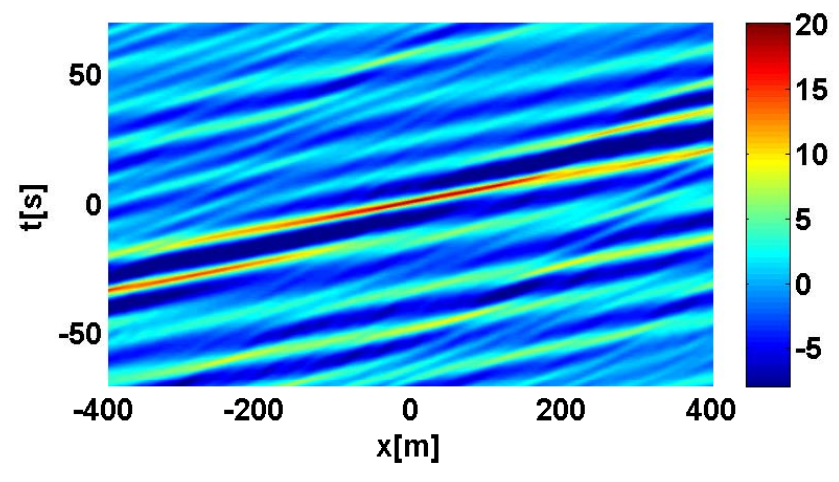

Fig. 14. The density of the elevation of Draupner wave using initial signal at $X_{\text {synth }}=-400 \mathrm{~m}$.

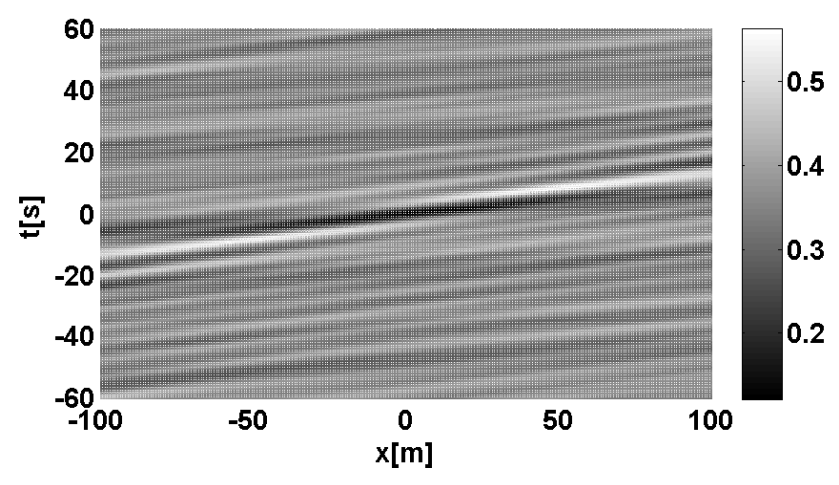

Fig. 15. The density plot of the variance of the total wave phase for the Draupner wave.

will predict the position, time and characteristics of the most extreme wave downstream and describe the extremal wave profile.

To predict the extreme wave, we compute the minimal value of the variance of the total wave phase; the density plot of the phase variance, $\operatorname{PV}(x, t)$ is shown in Fig. 17. In this case we computed the phase variance for frequencies between $\omega_{\min }=0.3$ and $\omega_{\max }=0.7$, and obtain $\mathrm{PV}_{\text {foc }}=0.22$ at $(5089.5 ; 1994)$. The calculated value of coherence is $\Gamma=0.78$, and the corresponding pseudo-maximal signal has $\alpha=0.81$ (scaling factor of 0.22 ). The pseudo-maximal signal has linear maximal amplitude of $4.30 \mathrm{~m}$. Adding the second order contributions, the maximal amplitude becomes $4.32 \mathrm{~m}$. For validation, we performed nonlinear simulations with the linear and the (nonlinear) AB-model of the complete time signal at positions downstream the observation point. For these numerical simulations we predicted the position and time of the highest wave as listed in Table 3.

The plots in Fig. 18 show the time signal of the elevation at the point of maximal amplitude as calculated by the nonlinear AB-model (solid), with superimposed on it (dashed) the profile of the pseudo-maximal wave as predicted by our prediction method but shifted in time some $5 \mathrm{~s}$ to let the crests 
Table 2. Parameters for the Draupner wave.

\begin{tabular}{|c|c|c|c|c|}
\hline $\begin{array}{l}C A S E \\
\text { Depth } \\
\text { Data position } X_{\text {synth }} \\
H_{S} \text { at } X_{\text {synth }}\end{array}$ & $\begin{array}{l}\text { Draupner w } \\
70 \mathrm{~m} \\
-400 \mathrm{~m} \\
12 \\
\text { Prediction }\end{array}$ & Simulc & & Meas \\
\hline Meas position & & linear & $\mathrm{AB}$ & 0 \\
\hline$X_{\text {foc }}$ & 9 & 3 & 3 & \\
\hline$T_{\text {foc }}$ & 1.1 & 0.47 & 1.3 & 0 \\
\hline$\Gamma$ (coherence) & 0.88 & 0.87 & 0.81 & 0.85 \\
\hline Max Crest height & 20.22 & 19.09 & 19.32 & 18.5 \\
\hline Max Waveheight & 27.42 & 26.5 & 28.28 & 25.6 \\
\hline$H_{S}$ at $X_{\text {foc }}$ & 11.64 & 11.9 & 13.5 & 11.92 \\
\hline BFI & 0.49 & 0.48 & 0.61 & 0.55 \\
\hline
\end{tabular}

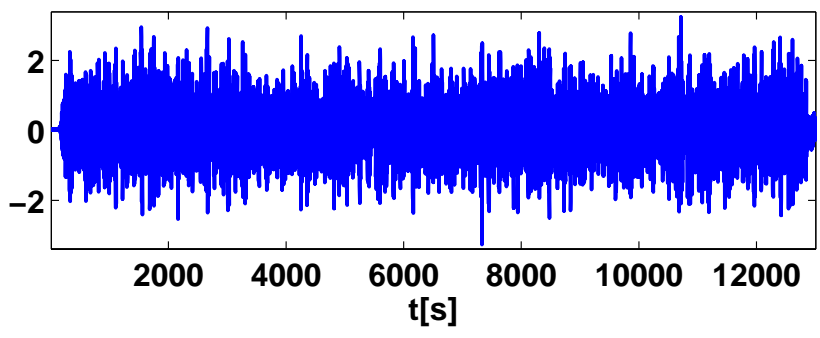

Fig. 16. Time signal of the irregular wave IW 12 at $1957.5 \mathrm{~m}$. This isa measured signal that will be used to forecast the freak wave downstream.

coincide. The predicted signal is also plotted in Fig. 18. Even though the maximal crest height of the pseudo-maximal signal is higher than the AB-simulation, it still describes the freak wave well around the highest crest. The results of the predicted position of the pseudo-maximal wave and the numerically simulated highest wave are presented in Table 3.

\subsubsection{Irregular wave IW9}

The second case of irregular wave has smaller period; $T_{p} \approx 9 \mathrm{~s}$. In this case we also use the time signal at $X_{\mathrm{obs}}=1957.5 \mathrm{~m}$ as initial signal for both prediction and ABsimulation. This initial signal is shown in Fig. 19.

The same strategy is executed to this initial signal to get the description and the prediction of a freak wave. Similar to the irregular wave IW12, this case is also approximated well by pseudo-maximal wave. According to the prediction, the coherence of the irregular wave IW9 is less than IW12. The maximal crest height of IW9 at focussing is a bit higher than the IW12, even though their significant wave heights at the observation point $X_{\mathrm{obs}}$ are almost the same.

In the minimization of the phase variance, we chose $\omega \in$ $[0.5 ; 1]$ for integration. Then the coherence of IW9 is $\Gamma \approx$ 0.72 at $x=2618.5$ and $t=8562$. The focussing position is

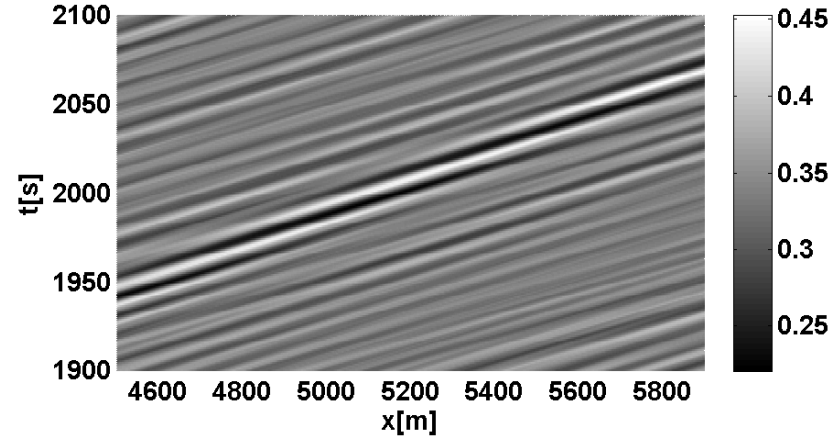

Fig. 17. The density plot of the variance of the total wave phase for IW12.

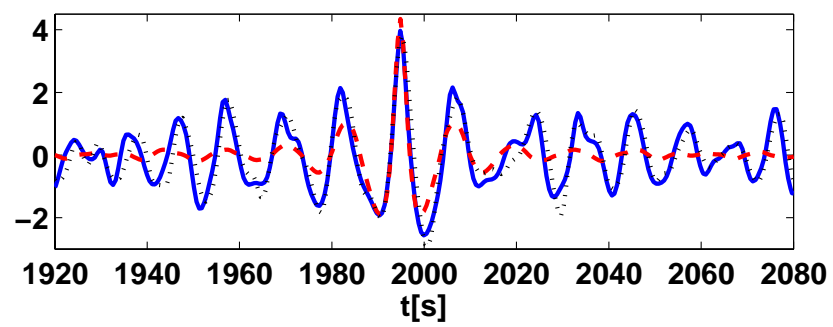

Fig. 18. Time signal as calculated by nonlinear $A B$ at focussing position (solid), nonlinear pm-wave predicted from time signal at $X_{\text {obs }}$ (dashed), and Signal prediction (dotted line) for IW12.

difficult to be identified in the density plot of the phase variance Fig. 20. The maximal wave at focussing by a nonlinear pseudo-maximal signal with scaling factor about 0.1 is shown in Fig. 21. We also compare it by the time signal at focussing computed by nonlinear AB-model. The parameters of the prediction and the AB-simulation are presented in Table 4. We do not have measurement data close to the focussing position, so for this case we only compare the prediction and the AB-simulation.

\section{Conclusions}

This paper has discussed the description and the predictability of extreme waves by investigating the phase coherence using the power spectrum and the phase information at a certain position. The extreme profile can be described in a small neighbourhood by a (pseudo-)maximal wave. Moreover, we have shown that the position and time of an extreme wave is predicted well by minimizing the variance of the total wave phase. It should be noted that this minimization requires the choice of a suitable frequency interval to which the variance is restricted, but that the precise choice is not yet well motivated.

Because of the symmetry in both linear and nonlinear evolution, extreme waves (in the linear and nonlinear maximal signal wave) appear at approximately the same position; except for some shift (in time and consistently in space) the 
Table 3. Parameters for irregular wave IW12.

\begin{tabular}{lllll}
\hline$C A S E$ & IW12 & & & \\
Depth & $30 \mathrm{~m}$ & & & \\
Data position $X_{\text {obs }}$ & $1957.5 \mathrm{~m}$ & & & \\
$H_{S}$ at $X_{\text {obs }}$ & $\begin{array}{l}\text { 3.14 } \\
\text { Prediction }\end{array}$ & Simulation & Meas \\
\hline Meas position & & linear & AB & 5106 \\
$X_{\text {foc }}$ & 5089.5 & 5112.5 & 5143.9 & \\
$T_{\text {foc }}$ & 1994 & 1995 & 1999 & 2004.8 \\
$\Gamma$ (coherence) & 0.78 & 0.76 & 0.78 & 0.76 \\
Max Crest height & 4.32 & 3.83 & 4 & 3.5 \\
Max Waveheight & 6.26 & 6.73 & 6.4 & 6.79 \\
$H_{S}$ at $X_{\text {foc }}$ & 3.14 & 3.1 & 3 & 3.2 \\
BFI & 0.17 & 0.19 & 0.17 & 0.16 \\
\hline
\end{tabular}

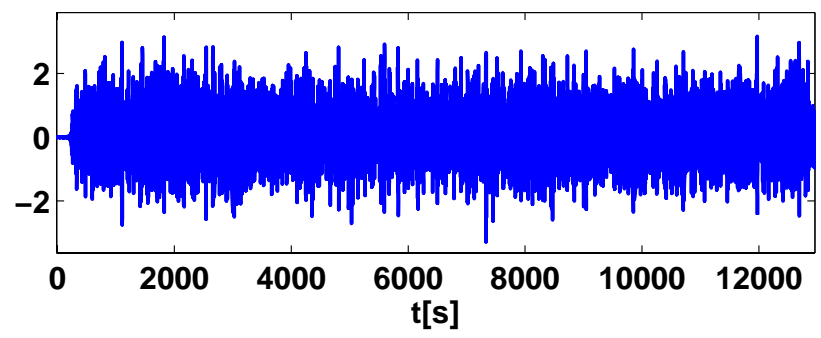

Fig. 19. Time signal of the irregular wave IW9 at $1957.5 \mathrm{~m}$ from the wave maker.

linear prediction gives a good estimation for the nonlinear evolution. In the four different applications, the focussing signal for which the phases are highly or moderately coherent could very well be modeled by a nonlinear maximal signal or by a pseudo-maximal signal; the parameters of the waves could be predicted to a good degree of accuracy from measurement data at a position upstream.

A final remark concerns the difference of the concept of pseudo-maximal wave with the concept of the New Wave model proposed by Walker et al. (2004); the (pseudo)maximal wave can be designed completely by knowledge of the spectrum, without the necessity as for the New Wave to determine the amplitude based on the probability of appearance.

\section{Appendix A}

\section{Stokes corrections}

Second order wave-wave interaction leads to nonlinear contribution as derived by Dalzell (1999). The form of second order solution is applied to define the nonlinear wave profile here. The final solution for the wave elevation, up to second

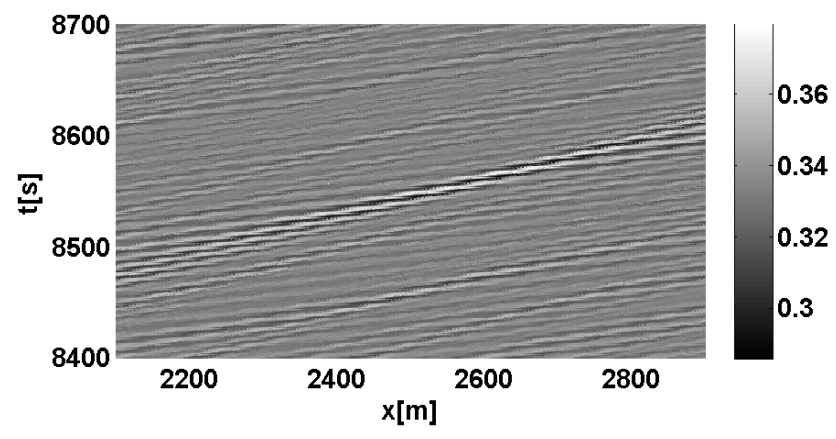

Fig. 20. The density plot of the variance of the total wave phase for IW9.

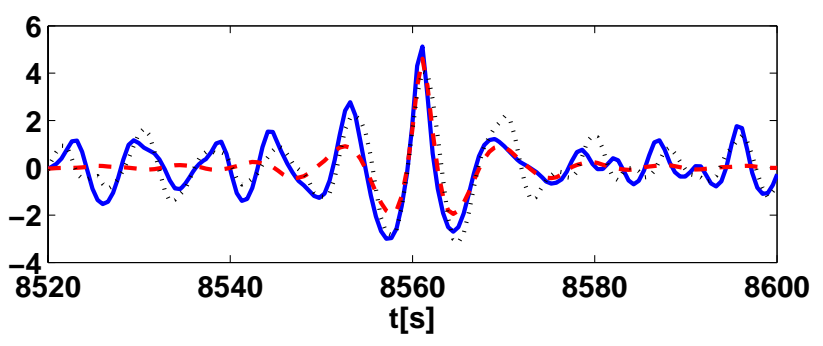

Fig. 21. Time signal as calculated by nonlinear $\mathrm{AB}$ at $X=$ $2626 \mathrm{~m}$ (solid), shifted nonlinear pm-signal predicted from time signal at $X_{\text {obs }}$ (dashed), and Signal prediction (dotted line) for IW9.

order, for the superposition of two waves is given by:

$$
\begin{aligned}
\eta(x, t) & =\sum_{j=1}^{2} a_{j} \cos \left(\varphi_{j}\right)+\sum_{j=1}^{2} a_{j}^{2} B_{0}\left(k_{j}\right) \\
& +\sum_{j=1}^{2} a_{j}^{2} B_{2}\left(k_{j}\right) \cos \left(2 \varphi_{j}\right)+a_{1} a_{2} B_{p}\left(k_{1}, k_{2}\right) \cos \left(\varphi_{1}\right. \\
& \left.+\varphi_{2}\right)+a_{1} a_{2} B_{m}\left(k_{1}, k_{2}\right) \cos \left(\varphi_{1}-\varphi_{2}\right)
\end{aligned}
$$

The first term of Eq. (A1) is the linear contribution. The rest are the second order contributions. The coefficients of the second order contributions depend on the wave number and frequency. These are defined by:

$$
\begin{aligned}
& B_{0}\left(k_{j}\right)=\frac{\left|k_{j}\right|}{4 \tanh \left(\left|k_{j}\right| h\right)}\left[2+\frac{3}{\sinh ^{2}\left(\left|k_{j}\right| h\right)}\right] \\
& B_{2}\left(k_{j}\right)=-\frac{\left|k_{j}\right|}{2 \sinh \left(2\left|k_{j}\right| h\right)}
\end{aligned}
$$


Table 4. Parameters for irregular wave IW9.

\begin{tabular}{llll}
\hline$C A S E$ & IW9 & & \\
Depth & $30 \mathrm{~m}$ & & \\
Data position $X_{\text {obs }}$ & $1957.5 \mathrm{~m}$ & & \\
$H_{S}$ at $X_{\text {obs }}$ & 3.1 & & \\
& Prediction & Simulation \\
\hline & & linear & $\mathrm{AB}$ \\
$X_{\text {foc }}$ & 2618.5 & 2478.5 & 2626 \\
$T_{\text {foc }}$ & 8562 & 8542 & 8560 \\
$\Gamma$ (coherence) & 0.72 & 0.71 & 0.73 \\
Max Crest height & $4.67(2$ nd order $)$ & 4.3 & 5.23 \\
Max Waveheight & $6.62(2 \mathrm{nd}$ order $)$ & 7.78 & 8.45 \\
$H_{S}$ at $X_{\text {foc }}$ & 3.10 & 3.18 & 3.05 \\
BFI & 0.27 & 0.18 & 0.21 \\
\hline
\end{tabular}

$B_{p}\left(k_{1}, k_{2}\right)=\frac{1}{2 g}\left[\omega_{1}^{2}+\omega_{2}^{2}-\omega_{1} \omega_{2}\left(1-P_{1}\right)\right.$

$\left.\cdot \frac{\left(\omega_{1}+\omega_{2}\right)^{2}+\Omega^{2}\left(\left|k_{1}+k_{2}\right|\right)}{\left(\omega_{1}+\omega_{2}\right)^{2}-\Omega^{2}\left(\left|k_{1}+k_{2}\right|\right)}+\frac{\left(\omega_{1}+\omega_{2}\right) P_{2}}{\left(\omega_{1}+\omega_{2}\right)^{2}-\Omega^{2}\left(\left|k_{1}+k_{2}\right|\right)}\right]$

$B_{m}\left(k_{1}, k_{2}\right)=\frac{1}{2 g}\left[\omega_{1}^{2}+\omega_{2}^{2}+\omega_{1} \omega_{2}\left(1+P_{1}\right)\right.$

$\left.\cdot \frac{\left(\omega_{1}-\omega_{2}\right)^{2}+\Omega^{2}\left(\left|k_{1}-k_{2}\right|\right)}{\left(\omega_{1}-\omega_{2}\right)^{2}-\Omega^{2}\left(\left|k_{1}-k_{2}\right|\right)}+\frac{\left(\omega_{1}+\omega_{2}\right) P_{2}}{\left(\omega_{1}-\omega_{2}\right)^{2}-\Omega^{2}\left(\left|k_{1}-k_{2}\right|\right)}\right]$

in which $j=1,2, \varphi_{j}=k_{j} x-\omega_{j} t$ is the phase, $a_{j}$ is the amplitude, $k_{j}=K\left(\omega_{j}\right)$ is wave number, $\omega_{j}$ is the frequency, and $h$ is water depth. For simplification we write

$$
\begin{aligned}
& P_{1}=\frac{1}{\tanh \left(\left|k_{1}\right| h\right) \tanh \left(\left|k_{2}\right| h\right)} \\
& P_{2}=\left[\frac{\omega_{1}^{3}}{\sinh ^{2}\left(\left|k_{1}\right| h\right)}+\frac{\omega_{2}^{3}}{\sinh ^{2}\left(\left|k_{2}\right| h\right)}\right] .
\end{aligned}
$$

The dispersion relation between $\omega_{j}$ and $k_{j}$ is given by $\omega_{j}^{2}=\Omega^{2}\left(k_{j}\right)=g\left|k_{j}\right| \tanh \left(\left|k_{j}\right| h\right)$.

Acknowledgements. We acknowledge Sverre Haver for providing the data of the Draupner Wave, and to MARIN Hydrodynamics Laboratory for the measurement data of a focussing signal and the irregular waves. This work was funded by the Netherlands Organization for Scientific Research, Technology foundation STW, number 7216 .

Edited by: A. Slunyaev

Reviewed by: two anonymous referees

\section{References}

Adcock, T. A. A. and Taylor, P. H.: The directional spreading of the Draupner wave and sea-state, in: Proc. 11th Int. Workshop on Wave Hindcasting and Forecasting, Halifax, Canada, 2009c.

Akhmediev, N., Ankiewicz, A., Soto-Crespo, J., and Dudley, J. M.: Rogue wave early warning through spectral measurements?, Phys. Lett. A, 375, 541-544, 2011.

Chen, X.: The set-down in the second-order Stokes' waves, in: International Conference on Hydrodynamics, Italy, 179-85, 2006.

Clamond, D. and Grue, J.: Interaction between envelope solitons as a model for freak wave formations, Part I: Long time interaction, C. R. Mecanique, 330, 575-580, 2002.

Dalzell, J.: A note on finite depth second order wave-wave interactions, Appl. Ocean Res., 21, 105-11, 1999.

Dysthe, K., Krogstad, H. E., and Muller, P.: Oceanic rogue waves, Annu. Rev. Fluid Mech., 40, 287-310, 2008.

Gemmrich, J. and Garrett, C.: Unexpected waves, J. Phys. Oceanogr., 38, 2330-2336, 2008.

Haver, S.: Freak Waves: A Suggested Definition and Possible Consequences for Marine Structures, in: Proceeding of Rogue Waves, 1-10, Brest, France, 2004.

Janssen, P.: Nonlinear four-wave interactions and freak waves, J. Phys. Oceanogr., 33, 863-884, 2003.

Kharif, C. and Pelinovsky, E.: Physical mechanisms of the rogue wave phenomenon, Eur. J. Mech. B.-Fluid., 22, 603-634, 2003.

Kharif, C., Phelinovsky, E., and Slunyaev, A.: Rogue waves in the Ocean, Advances in Geophysical and Environmental Mechanics and Mathematics, Springer-Verlag, Berlin Heidelberg, 2009.

Onorato, M., Osborne, A. R., and Serio, M.: Modulational Instability in Crossing Sea States: A Possible Mechanism for the Formation of Freak Waves, Phys. Rev. Lett., 96, 014503, doi:10.1103/PhysRevLett.96.014503, 2006.

Osborne, A., Onorato, M., and Serio, M.: Nonlinear Fourier analysis of deep water, random surface waves: Theoretical formulation and experimental observations of rogue waves, in: Proc. 14th Aha Huliko'a Winter Workshop, Honolulu, Hawaii, 2005.

Ross, S.: Introduction to Probability Models (9th Edition), Elsevier Inc., 79, 2007.

Shemer, L.: On Benjamin-Feir instability and evolution of a nonlinear wave with finite-amplitude sidebands, Nat. Hazards Earth Syst. Sci., 10, 2421-2427, doi:10.5194/nhess-10-2421-2010, 2010.

Shemer, L. and Sergeeva, A.: An experimental study of spatial evolution of statistical parameters in a unidirectional narrowbanded random wavefield, J. Geophys. Res., 114, C01015, doi:10.1029/2008JC005077, 2009.

Shemer, L., Sergeeva, A., and Liberzon, D.: Effect of the initial spectrum on the spatial evolution of statistics of unidirectional nonlinear random waves, J. Geophys. Res., 115, C12039, doi:10.1029/2010JC006326, 2010.

Shukla, P. K., Marklund, M., and Stenflo, L.: Modulational Instability of Nonlinearly Interacting Incoherent Sea States, JETP Letters, 84, 645-649, 2006.

Slunyaev, A.: Nonlinear analysis and simulations of measured freak wave time series, Eur. J. Mech.-B/Fluid., 25, 621-635, 2006.

Slunyaev, A., Pelinovsky, E., and Soares, C. G.: Modeling freak waves from the North Sea, Appl. Ocean Res., 27, 12-22, 2005.

van Groesen, E. and Andonowati: Variational derivation of KdVtype of models for surface water waves, Phys. Lett. A, 366, 195- 
201, 2007

van Groesen, E. and Andonowati: Fully dispersive dynamic models for surface water waves above varying bottom, Part 1: Model equations, Wave Motion, 48, 658-667, 2011.

van Groesen, E. and van der Kroon, I.: Fully dispersive dynamic models for surface water waves above varying bottom, Part 2: Hybrid spatial-spectral implementations, Wave Motion, 49, 198 $211,2012$.

van Groesen, E., Andonowati, Liam, L. S., and Lakhturov, I.: Accurate modelling of unidirectional surface waves, J. Comput. Appl. Math., 234, 1747-1756, 2010. van Groesen, E., Bunnik, T., and Andonowati: Surface wave modelling and simulation for wave tanks and coastal areas, International Conference on Developments in Marine CFD,Chennai, India, RINA, 59-63, 2011.

Walker, D. A. G., Taylor, P. H., and Taylor, R. E.: The shape of large surface waves on the open sea and the Draupner New Year wave, Appl. Ocean Res., 26, 73-83, 2004. 